\title{
Nonlinear mechanisms of sound generation in a perturbed parallel jet flow
}

\author{
By N. D. SANDHAM ${ }^{1}$, C. L. MORFEY AND Z. W. HU ${ }^{1}$ \\ ${ }^{1}$ School of Engineering Sciences, University of Southampton, Southampton SO17 1BJ, UK \\ ${ }^{2}$ ISVR, University of Southampton, Southampton SO17 1BJ, UK
}

(Received 29 September 2005 and in revised form 15 March 2006)

An initial value problem with relevance to jet noise is investigated. A plane parallel jet flow is subjected to a spatially localized initial disturbance and is then left to evolve according to the two-dimensional compressible Navier-Stokes equations. The hydrodynamic response is in the form of a convecting vortex packet. The Ffowcs WilliamsHawkings approach is formulated in the time domain and used to extrapolate from the simulated near field to the acoustic far field. The predominant downstream sound radiation comes from an early stage of nonlinear development of the vortex packet. Two simplified models to account for the radiation are introduced, based on nonlinear mode interactions on a prescribed base flow. The first uses two sets of linearized Euler equations, coupled via the inviscid Lilley-Goldstein acoustic analogy. This formulation separates the linear sound field from the sound field driven by nonlinear interactions; qualitative agreement of the latter with the Navier-Stokes computations demonstrates the importance of nonlinear interactions. The second model uses combinations of linear inviscid eigenmodes to drive the sound field, which allows extraction of the dominant mode interactions responsible for the observed radiation pattern. The results indicate that a difference-wavenumber nonlinear interaction mechanism dominates sound radiation from subsonic instability modes in shear flows.

\section{Introduction}

The noise from jet engines remains a significant environmental problem. Highfrequency sound is usually attributed to the turbulent shear layers around a potential core during the early development of the jet while low frequencies are attributed to the downstream jet evolution. The principal method to reduce jet noise over the past half-century has been the use of progressively higher bypass ratio engines. However, further development in this direction is at the cost of propulsive efficiency and recent efforts have been concentrated more on nozzle design for which accurate prediction methods are needed.

A major limitation on the accuracy of predictions of sound produced by turbulent flows is the representation of the turbulence. Scaling arguments, such as the Lighthill (1952) $M^{5}$ law for the efficiency of conversion of jet power into sound power, require only the assumption that the unsteady turbulence stresses scale as the jet velocity squared. However to go further and predict sound spectra, or develop control schemes to reduce noise, requires a more complete understanding of turbulence that continues to elude theory. With the growth of computer power it has become feasible to couple the theoretical formulations of aeroacoustics with numerical simulations of turbulent jets, at least for simple nozzle geometry (see Shur, Spalart \& Strelets 2005 for a 


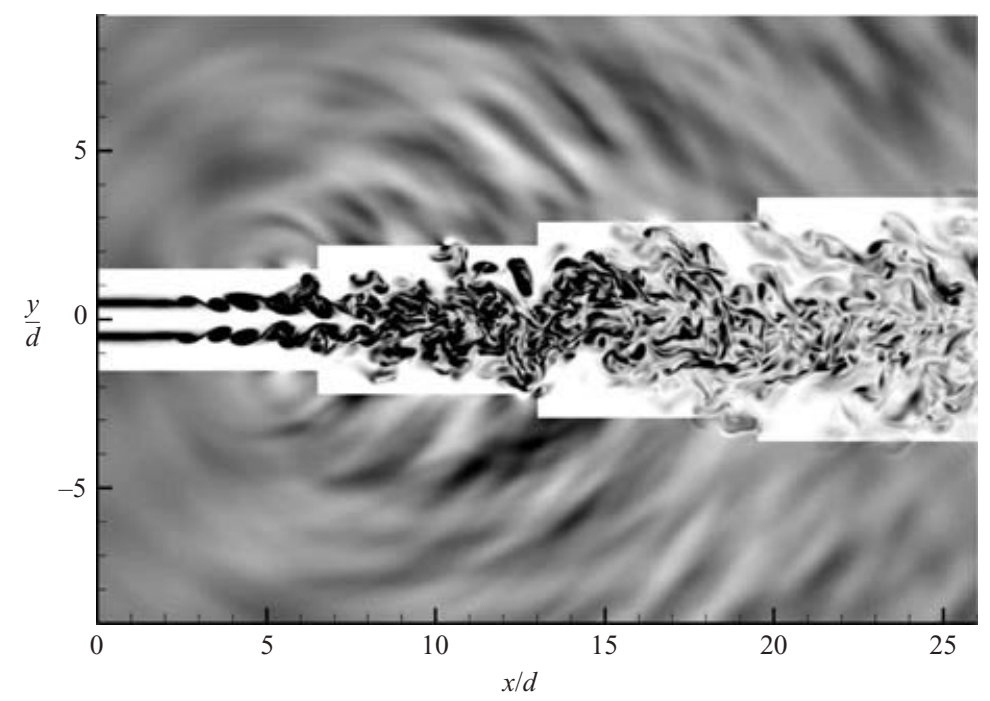

FIGURE 1. Direct simulation of jet noise from a plane jet with transition to turbulence $(R e=2000$ based on jet width). The inner region shows contours of vorticity magnitude and the outer region shows contours of dilatation rate.

review of some of the practical issues). Direct computations of sound (see e.g. Freund 2001) solve the compressible Navier-Stokes equations in a finite domain that includes the near sound field. With this approach care needs to be taken to minimize the reflections of waves from the outer boundaries of the computational domain; for a recent review see Colonius (2004). To predict the acoustic far field, particularly from three-dimensional calculations, computational cost usually dictates the use of cheaper methods such as linearized Euler numerical matching to a wave equation solution domain, or surface integral methods such as the Kirchhoff or Ffowcs Williams \& Hawkings (1969 hereafter denoted FW-H) approaches.

\subsection{Large-scale structures, instability waves and sound radiation}

An example of a direct computation of sound is given on figure 1. This shows vorticity (inner region) and dilatation rate (outer region) for a Mach 0.9 plane jet at Reynolds number 2000 (based on jet width), computed using the method of $\mathrm{Hu}$, Morfey \& Sandham (2002). The sound field emanates from a region of the flow (centred around $x=5$ jet widths) that is dominated by large-scale organized structures. Such structures in free shear flows (cf. Brown \& Roshko 1974) have properties, such as convection speeds, in common with instability waves developing on a similar base flow and there is a direct connection between instability wave growth rates and shear layer spreading rate (e.g. Morris, Giridharan \& Lilley 1990; Sandham \& Reynolds 1990). Recent experimental work has provided evidence that instability waves are present in fully turbulent jets at high Reynolds number: Suzuki \& Colonius (2006) used a nearfield microphone array to extract the instability-wave pressure signature and found remarkably good agreement with linear theory. A model for the production of sound based on linear instability waves was developed by Tam \& Morris (1980), assuming a slowly spreading base flow, which resulted in the spatial growth and decay of linear eigenmodes. The approach has been of most use in supersonic flows, where the instability waves responsible for the sound radiation are amongst the most unstable. 
A related model for subsonic jets, based on convected waves with a prescribed amplitude distribution, was analysed by Crighton \& Huerre (1990) in the asymptotic limit of slow amplitude variation compared to wavelength. It gave a superdirective radiation pattern, which replicated some earlier subsonic jet experiments of Laufer \& Yen (1983) and has since been observed in several numerical studies of unsteady laminar shear flows, forced so as to produce vortex pairing. For example, Colonius, Lele \& Moin (1997) imposed disturbances on a two-dimensional plane mixing layer at the fundamental (most unstable) frequency and two subharmonics. Although the principal sound radiation appeared to originate from the two vortex pairing locations in the flow, its directivity was consistent with a convected wavepacket model similar to that of Crighton \& Huerre (1990), with an asymmetric envelope shape. Mitchell, Lele \& Moin (1999) conducted a similar study for an axisymmetric jet over a range of Mach numbers (from 0.4 to 1.2). They found superdirective radiation patterns at higher Mach numbers, while at low Mach numbers (where the source region is acoustically compact in the streamwise direction) there was a lobed directivity pattern similar to that of a compact axisymmetric quadrupole.

\subsection{Simplified physical model and objectives}

Direct numerical simulations of compressible turbulent flow contain complete information about the turbulence and the near acoustic field, and one approach to noise research is to probe the simulation databases to try and understand the mechanisms of sound production, with the hope that the improved understanding will lead to better physical modelling. A complementary approach is to develop simplified model problems that contain the main physical mechanisms. Here, we follow the latter approach and focus on the transient response of a parallel plane jet to a point disturbance input. A conceptually similar problem was studied by Howe (1970) in the context of sound transmission across a vortex sheet; but here we are able to compute the full flow field exactly, including the shear layer unstable response. This simplified model problem focuses attention on vortex interactions during temporal evolution of a perturbed parallel flow, similar in velocity profile to the mean flow field of a plane turbulent jet (as in figure 1) near the end of the jet potential core. In contrast to spatial simulations, where time-dependent disturbances are imposed at an inflow boundary, the present model uses a large (spatially periodic) domain in the streamwise direction, forced by a localized initial condition. This allows the origin of particular sound waves to be clearly located.

The objective of the present work is to distinguish between a linear and nonlinear source mechanism. A simplified nonlinear model is formulated by involving the second term of an amplitude expansion of the perturbation. The success of this model, compared to the strictly linear response, suggests a new interpretation of the origins of jet noise in terms of difference-mode interactions.

The two main sections that follow deal respectively with the Navier-Stokes simulations and the simplified models. In $\S 2$ the problem is specified and results for the near and far sound field are presented. A base flow is also extracted from the simulation for use in the subsequent modelling. In $\S 3$, two models are proposed, one based on the linearized Euler equations $(\S 3.1)$, the other on eigenmode-forcing $(\$ 3.2)$. Consistency between the approaches is demonstrated; this allows the identification of nonlinear eigenmode interactions as significant contributors to the sound radiation. Implications are discussed in $\S 4$. 


\section{Impulsive response of a parallel plane jet}

\subsection{Problem specification}

We consider an initially parallel two-dimensional jet flow with a rounded top-hat velocity profile:

$$
u_{0}=\frac{1}{2}\left(\tanh \left(y+y_{0}\right)-\tanh \left(y-y_{0}\right)\right)
$$

with $y_{0}=5$. All variables are expressed in dimensionless form with length scaled on one half of the initial shear layer vorticity thickness (so that $\delta_{\omega}=2$ ). The Reynolds number based on the initial jet width is $R e_{J}=2 y_{0}^{*} U_{J}^{*} / \nu_{J}^{*}=2000$, where an asterisk denotes dimensional variables and subscript $J$ denotes a jet potential-core reference condition (note that the choice of $y_{0}=5$ is large enough that this is effectively the same as the jet centreline condition at time $t=0$ ). The governing equation and numerical method are given in Appendix A.

The initial jet potential-core temperature is taken equal to the ambient fluid temperature and the initial temperature distribution (normalized as $T=T^{*} / T_{J}^{*}$ ) is obtained from a Crocco-Busemann relation

$$
T_{0}=1+\frac{\gamma-1}{2} M^{2} u_{0}\left(1-u_{0}\right)
$$

where $M$ is the jet Mach number (the initial ratio of jet potential core velocity $U_{J}^{*}$ to sound speed $c_{J}^{*}$ ). For $M=0.9$ this leads to a small (4\% maximum) temperature rise in the shear layer. The model calculations in $\S 3$ use a constant-temperature base flow.

The jet is perturbed initially by a localized two-dimensional disturbance to the normal component of velocity, centred in the upper shear layer at $x=x_{0}$ :

$$
v_{0}(x, y)=a_{0} \tanh \left(x-x_{0}\right) \exp \left(-\left(x-x_{0}\right)^{2}\right) \exp \left(-b_{0}\left(y-y_{0}\right)^{2}\right) .
$$

The disturbance shape is resolved on the computational grid. We take $x_{0}=60$, $a_{0}=0.05$ and $b_{0}=0.1$ and then follow the temporal evolution of what we shall call the 'vortex packet' and the sound emitted from it.

\subsection{Vortex packet and near-field sound radiation}

We first present some results from a large-box simulation, with box lengths $L_{x}=400$, $L_{y}=300$ on a grid with $1024 \times 801$ grid points. A typical result from the late stages of the simulation is shown on figure 2 by dilatation rate and vorticity contours at $t=293.7$. The dilatation rate plot (figure $2 a$ ) shows a number of sound waves that had their genesis in the early nonlinear evolution of the wavepacket and are now travelling predominantly in the downstream direction. The leading wave is an expansion wave that has just re-emerged at the left-hand boundary due to the periodic boundary condition. Successive expansion and compression waves are visible and more waves emerge during later evolution of the vortex packet. The vorticity plot (figure $2 b$ ) shows the nonlinear vortex packet that has developed from the jet instability triggered by the forcing. The vortex packet is convecting downstream and spreading, as expected in a convectively unstable flow. A $t-x$ diagram is shown on figure 3 , constructed by tracking the locations of all local pressure minima in the flow. The leading edge of the vortex packet is moving at approximately the jet core velocity while the trailing edge moves at $12 \%$ of the jet core velocity. The envelope of the vortex packet is shown on figure 3 by the lines $x=32+t$ and $x=97+0.12(t-65)$. Beyond $t \approx 250$ the trajectories become noticeably curved, indicating strong acceleration or deceleration of vortices as they move under their mutual induction. 

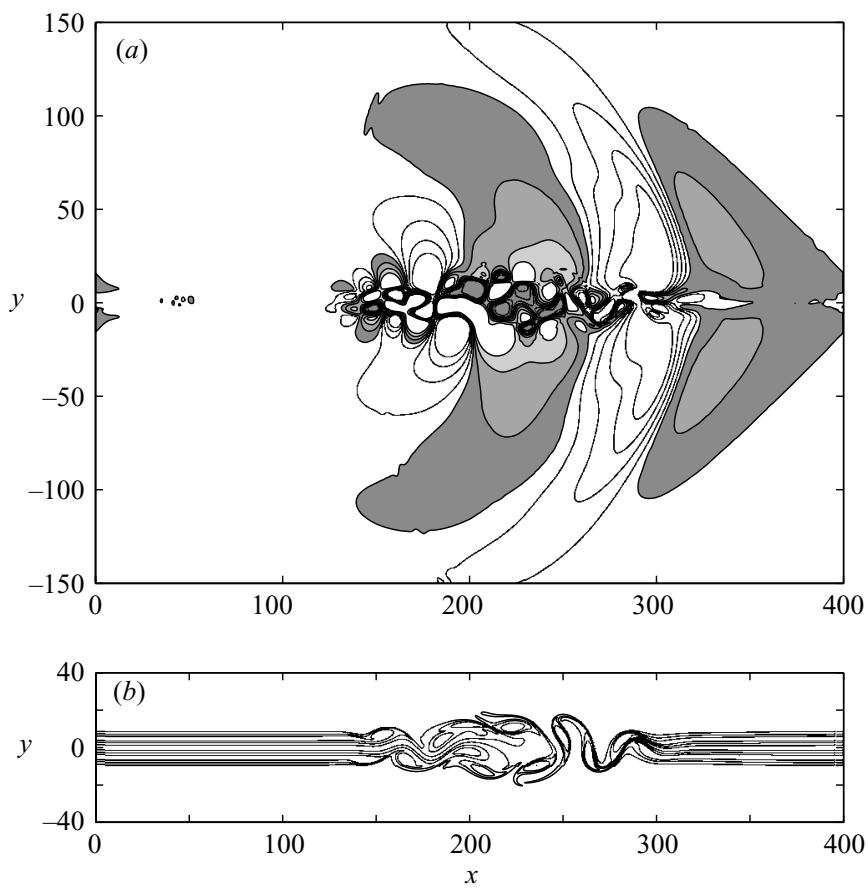

Figure 2. Contours of $(a)$ dilatation rate (contour levels $\pm[0.05,0.20,0.40,0.65] / 300$; filled contours are used for positive values, corresponding to areas of expansion) and $(b)$ vorticity ( 8 equally spaced contours) at $t=293.7$ showing the sound waves being emitted by the vortex packet.

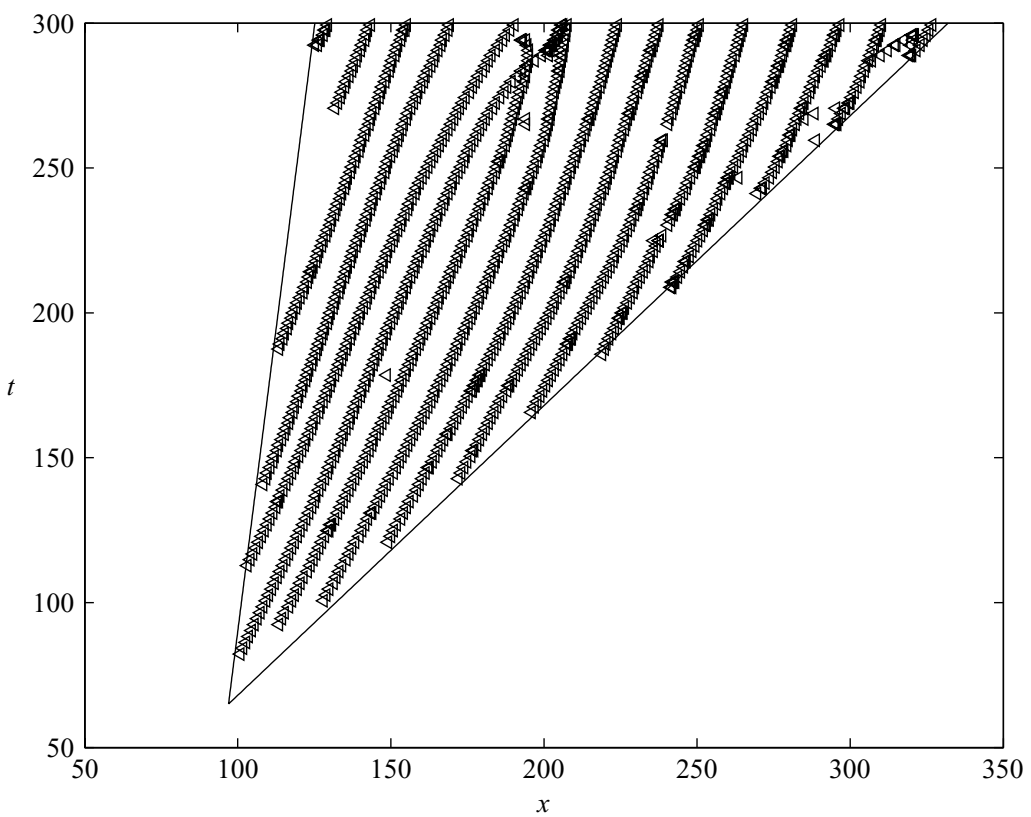

FIGURE 3. A $t-x$ diagram showing the convection of vortices, located by local pressure minima. 


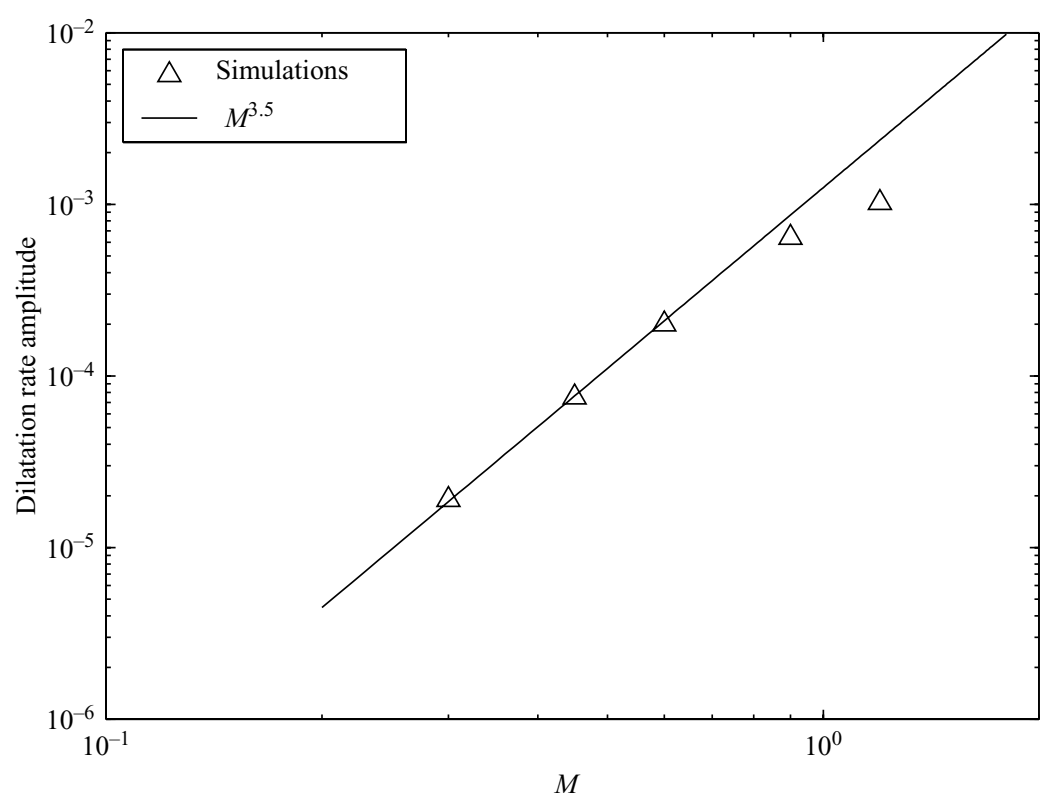

FIGURE 4. Variation in dilatation rate amplitude of emitted sound wave with Mach number.

Changing the Mach number affects the amplitude of the emitted sound. Figure 4 shows dilatation rate amplitudes from a series of small-box simulations at jet Mach numbers of $0.3,0.45,0.6,0.9$ and 1.2 , with the forcing initially in the lower shear layer. The amplitude is computed at $x=210, y=-78$ as half the expansion-to-compression variation during the first sound wave passage following the vortex evolution. The transverse location is approximately $\lambda / 2$ (where $\lambda$ is the acoustic wavelength) away from the jet centreline. A low-Mach-number scaling law based on compact quadrupole sources is also shown on the figure as a line proportional to $M^{3.5}$. The exponent of 3.5 comes from the two-dimensional Green function for a quadrupole source, which yields the scaling exponent of far-field pressure fluctuations as 1.5, together with a further exponent of 2 to convert from pressure to dilatation rate. The asymptotic prediction matches the simulation results rather well at low Mach numbers, lending support to the use of this simplified configuration to study the mechanisms of jet noise production.

\subsection{Extrapolation of the acoustic field}

So far only the near acoustic field has been studied. Since we later focus on simplified models to predict the near-field sound, it is important to check at this stage that such sound does indeed radiate out into the far field, and to investigate the structure of this far-field radiation. In spatially developing jet problems the $\mathrm{FW}-\mathrm{H}$ method is complicated by jet structures crossing a downstream surface and leading to spurious sound radiation (Shur et al. 2005). Here, due to the initial value nature of the problem, this complication is absent and other aspects of the performance of the FW-H method can be assessed. A time-domain solution technique is adopted.

Following the FW-H approach, the solution of a linear wave equation (with operator $\mathrm{L}=c_{\infty}^{-2} \partial^{2} / \partial t^{2}-\partial^{2} / \partial x_{i}^{2}, c_{\infty}$ being the sound speed of the ambient medium) 


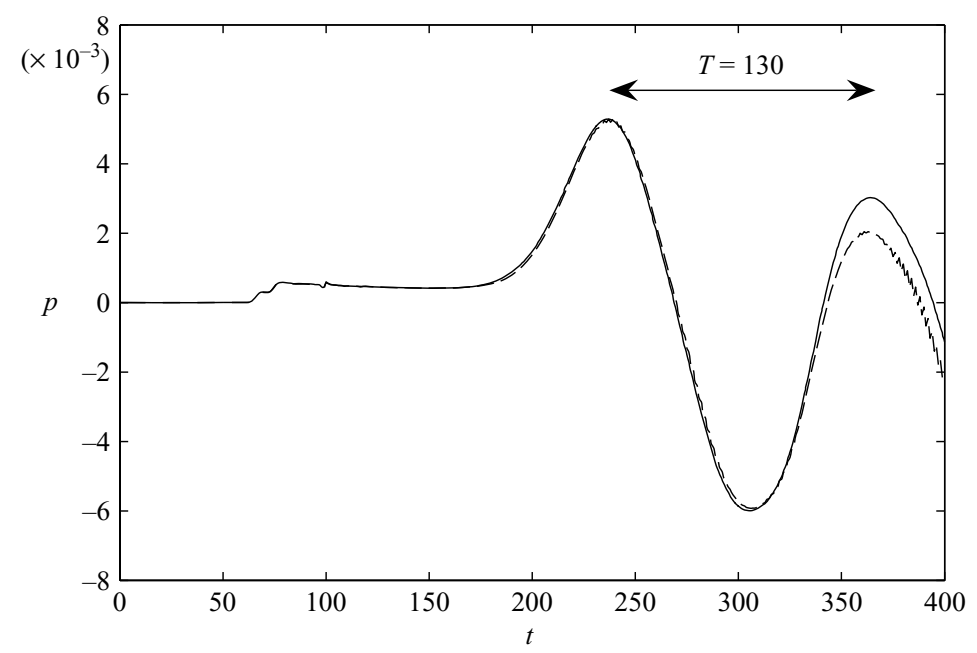

FIGURE 5. Evaluation of the FW-H surface method. Comparison of FW-H prediction for the pressure perturbation (dashed line) with simulation result (solid line) at $(x=150, y=-78)$. The peak-to-peak period of the oscillation is $T \approx 130$.

in a volume $V$ bounded by a surface $S$ is given by

$$
p(\boldsymbol{x}, t)=\int_{-\infty}^{\infty} \int_{S} R(\boldsymbol{y}, \tau) g(\boldsymbol{x}, t ; \boldsymbol{y}, \tau) \mathrm{d}^{2} \boldsymbol{y} \mathrm{d} \tau .
$$

Here, we take the volume $V$ to be a region outside the jet bounded by the plane $y=y_{F W-H}$, where $y_{F W-H}$ is taken as a constant. The observer is at a location $\boldsymbol{x}$ and time $t$ within $V$ and the integration is carried out over the bounding plane $S$, for all surface locations $\boldsymbol{y}$ and all source times $\tau$. The surface integral is not extended to the 'image' surfaces represented by the periodic boundary conditions in the $x$-direction, since we are interested in the far-field radiation from an individual wavepacket. Assuming (i) that there are no sources within the volume $V$, (ii) that the surface $y=y_{F W-H}$ is in the acoustic region, and (iii) that viscous terms can be neglected, a simplified expression for $R(\boldsymbol{y}, \tau)$ is obtained (see e.g. FW-H; Lockard 2000; Hu, Morfey \& Sandham 2003):

$$
R(\boldsymbol{y}, \tau)=-\frac{\partial\left(\rho_{\infty} u v\right)}{\partial x}-\frac{\partial\left(\rho_{\infty} u u+p\right)}{\partial y}+\frac{\partial\left(\rho_{\infty} v\right)}{\partial \tau} .
$$

The relevant Green function (see e.g. Crighton 1975) is

$$
g(\boldsymbol{x}, t ; \boldsymbol{y}, \tau)=\frac{H\left(t-\tau-r / c_{\infty}\right)}{2 \pi\left[(t-\tau)^{2}-\left(r / c_{\infty}\right)^{2}\right]^{0.5}},
$$

where $r=|\boldsymbol{x}-\boldsymbol{y}|$ is the distance from the source to the observer. The Green function contains an integrable singularity, which can be treated without special numerical considerations: a rectangular integration method was applied in this work. The numerator of the Green function is the standard Heaviside function.

As a basic test, a surface was located at $y_{\mathrm{FW}-\mathrm{H}}=-39$ and used to predict the pressure at a point $(x=150, y=-78)$, which is still within the simulation domain so that the Navier-Stokes solution is available for comparison. The simulation was run with $L_{x}=480$ up to time $t=400$ and source data were accumulated. Figure 5 shows the $\mathrm{FW}-\mathrm{H}$ prediction (dashed line) compared with the numerical simulation. It can be seen that there is very good agreement, even during the initial development at 


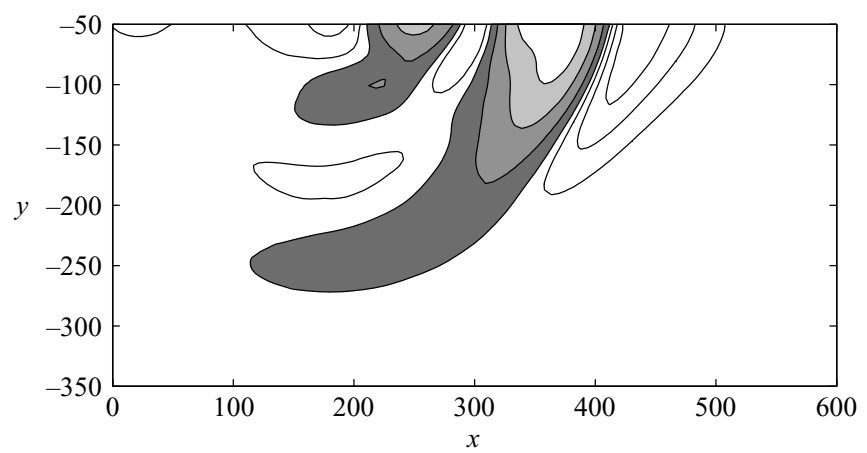

FIGURE 6. Prediction of pressure field at $t=400$ from FW-H surface method. Contour levels $\pm[0.05,0.20,0.40,0.65] / 20$; filled contours are used for positive pressure perturbations.

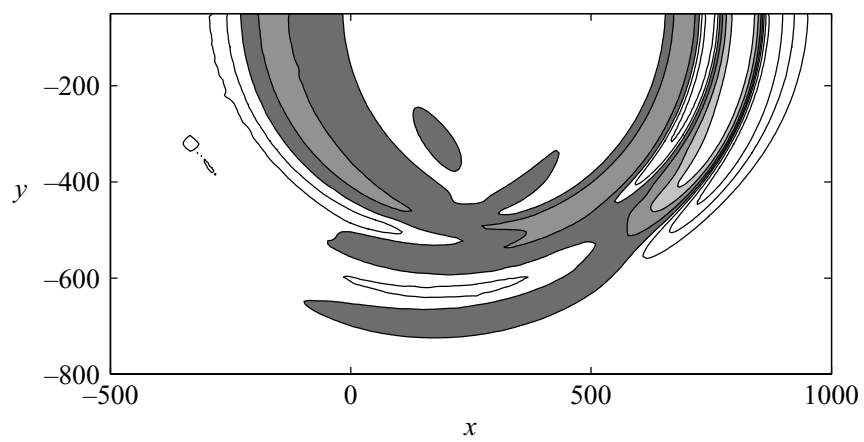

FIGURE 7. Prediction of pressure field at $t=800$ from FW-H surface method. Contour levels $\pm[0.05,0.20,0.40,0.65] / 50$; filled contours are used for positive pressure perturbations. Note that a larger domain is used compared to figure 6.

quite low amplitude. The initial response is in the form of two steps (at $t=60$ and $t=70$ ) due to the initial adjustment of the mean profile (since (2.1) is not a solution of the Navier-Stokes equations), and a small pulse at $t=100$ when the acoustic response to the initial disturbance has reached the observer location. The deviations at $t \geqslant 350$ may be due to volume source terms, not included in the prediction, or due to boundary effects.

Having been validated, the $\mathrm{FW}-\mathrm{H}$ procedure can be applied to extrapolate the acoustic field beyond the original simulation box. Figure 6 shows the pressure field computed for the region $0 \leqslant x \leqslant 600$ and $-350 \leqslant y \leqslant-50$ at time $t=400$; this is a later evolution of the simulation field shown earlier (figure 2). At a still later time $t=800$, but still using source data for $0 \leqslant \tau \leqslant 400$, the acoustic field for $0 \leqslant x \leqslant 1000$ and $-800 \leqslant y \leqslant-50$ is shown on figure 7 . It can be seen that the jet evolution up to $\tau=400$ results in a sound packet that propagates outwards. In contrast to figure 6 , figure 7 shows low-level sound radiation in the upstream direction which originates at a later stage of vortex packet development than the initial (much stronger) downstream radiation. A directivity plot of the logarithmic quantity

$$
P=10 \log _{10} \int_{0}^{t} p^{2} \mathrm{~d} t
$$




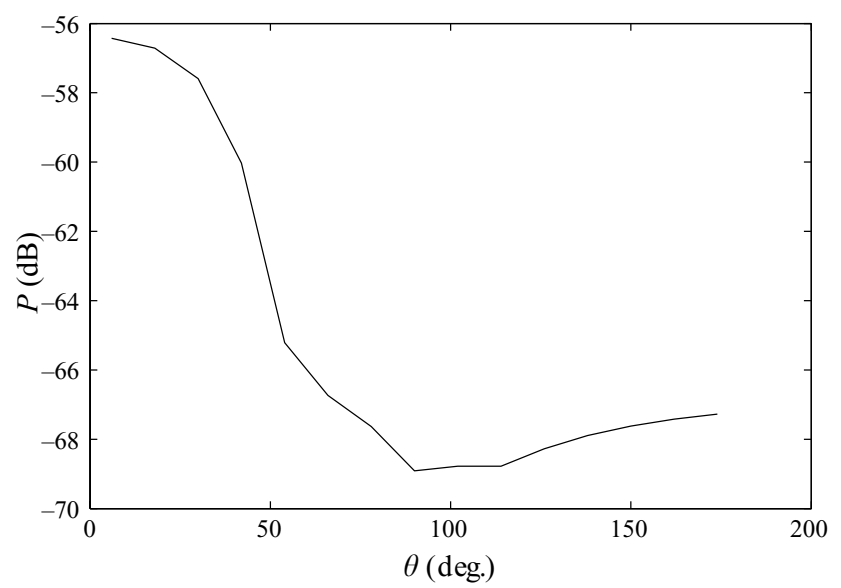

FiguRE 8. Far-field directivity from FW-H extrapolation of the Navier-Stokes simulation (jet Mach number $M=0.9$, distance $r=600$; angles are measured from the downstream direction).

in the acoustic far field is shown on figure 8. For this plot the square of the pressure was integrated to large time $t$ for an arc of observer positions, located a distance of 6000 (600 jet widths) from the origin of the disturbance at $x_{0}=60$. The difference in $P$ between the downstream and upstream sound radiation is $12-14 \mathrm{~dB}$. Note that there is significant radiation of sound in the direction $\theta<30^{\circ}$ : in this respect the sound field is similar to the pairing-generated noise seen in Colonius et al. (1997) and to the low-Strouhal-number measurements of Lush (1971) (here $S t \approx 0.1$ based on a wave period of $T \approx 100$ ). There is no cone of silence since the acoustic wavelength is an order of magnitude larger than the shear layer thickness (Tester \& Morfey 1976).

\subsection{Flow structures and mean flow}

The origin of the main emission of sound can be traced back to the early evolution of the vortex packet. Figure 9 shows dilatation rate $(a)$ and vorticity $(b)$ contours at $t=175.5$ : the emerging waves are visible in the outermost contours of dilatation rate. From the vorticity plot it can be seen that this is an early stage of nonlinear roll-up, just as the vortices are being displaced up and down slightly by their mutual interaction; earlier evolution is limited to growth of the vortices. Around each vortex the dilatation rate has a quadrant structure. The quadrants located towards the oncoming flow (in a frame of reference moving with the vortex) have positive dilatation rate corresponding to expansion; in the other two quadrants the dilatation rate is negative. Our aim in $\S 3$ will be to synthesize the dilatation rate of figure 9 from nonlinear interactions of instability waves on a selected base flow.

To determine the base flow, we took a streamwise average of $u$ within the vortex packet and fitted it with an analytic profile of the form

$$
\bar{u}(y)=\frac{1}{2}\left(\tanh \left(\frac{y+y_{0}}{\delta}\right)-\tanh \left(\frac{y-y_{0}}{\delta}\right)\right),
$$

which for $\delta=1$ recovers the initial condition (2.1) used in the simulation, and for increasing $\delta$ changes the velocity profile towards a fully developed jet. Figure 10 shows a comparison between the model profiles from (2.8) and the Navier-Stokes profiles, obtained by averaging over a selected range of $x$ within the vortex packet envelope shown on figure 3 . Values of $\delta$ were derived by minimizing the squared deviation between the simulation and model profiles. It can be seen that the averaged 

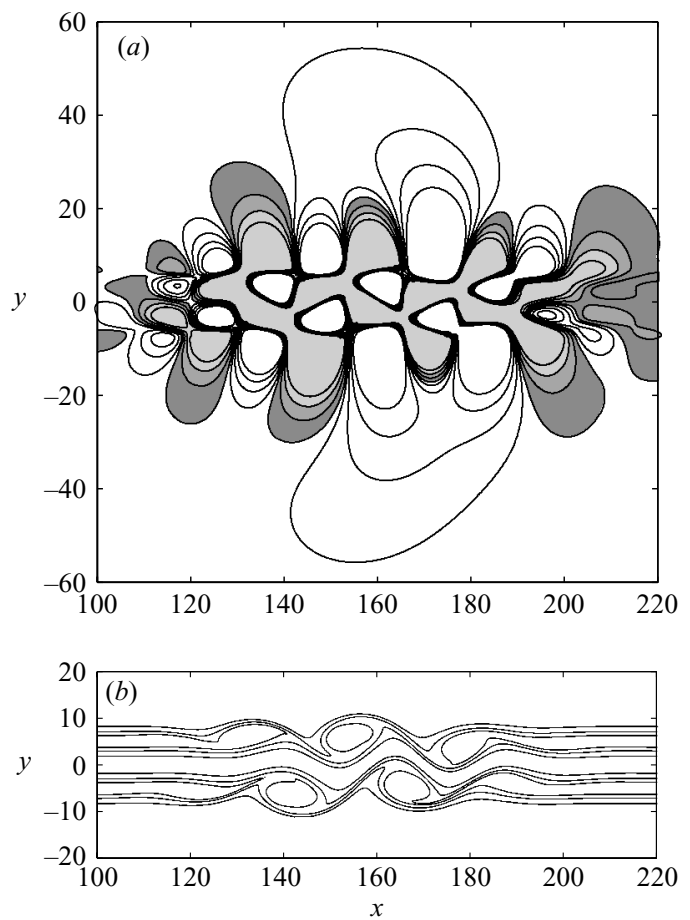

FIGURE 9. Flow structure at $t=175.5$ as sound is first emitted: $(a)$ dilatation rate (contour levels $\pm[0.05,0.20,0.40,0.65] / 600$; filled contours are used for positive values, corresponding to areas of expansion), (b) vorticity (8 equally spaced contours).

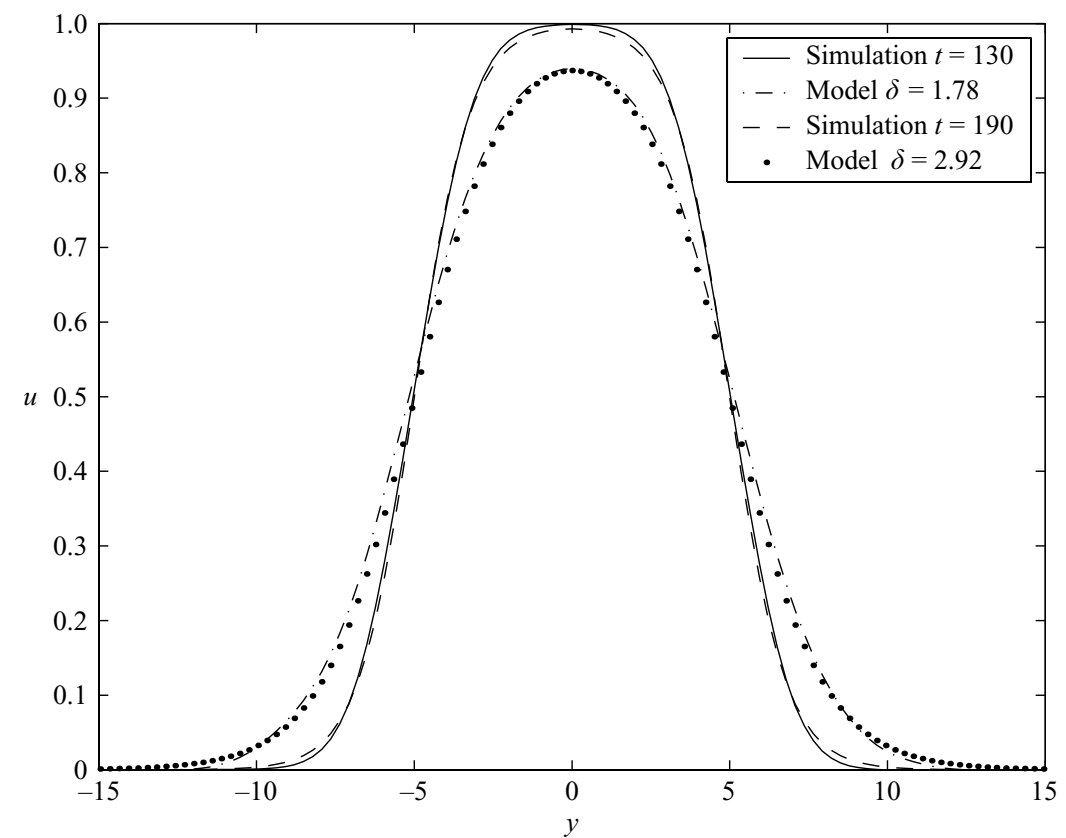

FIGURE 10. Comparison between velocity profiles from equation (2.8) and averages over the whole vortex packet from the numerical simulation, at two different times. 


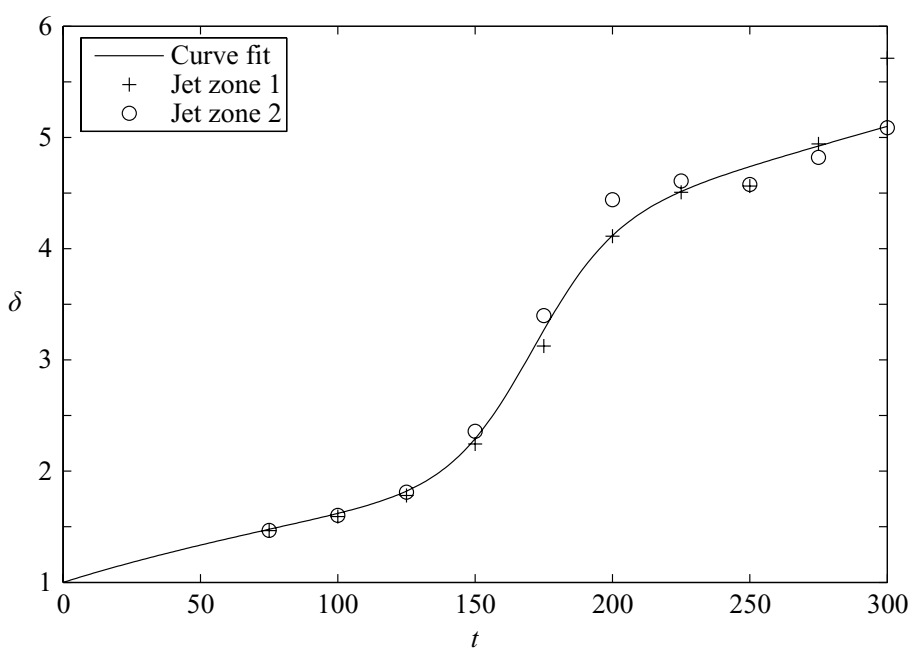

FIGURE 11. Variation of $\delta$ during the early evolution of the vortex packet. The symbols are extracted from the Navier-Stokes solutions using a best fit to the model profile of the actual profiles in the inner region of the vortex packet. The solid line is an analytic curve fit through the symbols given by (2.9).

simulation profiles based on the whole vortex packet are fitted well by the model equation. Finally the time variation of $\delta$ is shown on figure 11, where the two symbols denote averages over two sub-regions of the vortex packet: zone 1 is the inner $50 \%$, and zone 2 is the inner $25 \%$, of the vortex packet region shown on the $x-t$ diagram (figure 3). The solid line is a fit through these data of the form:

$$
\delta=c_{3}(1-f) \sqrt{t+\frac{1}{c_{3}^{2}}}+f\left(c_{4}+c_{5} t\right)
$$

with

$$
f=0.5\left(1+\tanh \left(\frac{t-c_{1}}{c_{2}}\right)\right) .
$$

The constants are $\left(c_{1}, c_{2}, c_{3}, c_{4}, c_{5}\right)=(170,30,0.125,3,0.007)$. It can be seen that there is a rapid change in $\delta$ for $150<t<200$; profiles in this region correspond to the end of the potential core in the plane jet seen in figure 1 .

\section{Linear and nonlinear modelling}

In this section we propose a method of computing the sound radiation from nonlinear mode interactions that yields qualitative agreement of the near-field sound with the Navier-Stokes computations of $\S 2$. We start with a coupled linearized Euler model and then simplify this further to a model based on linear stability eigenmodes.

\subsection{Coupled linearized-Euler model}

The nonlinear model consists of a pair of linearized Euler problems, where the second depends on the output of the first (one-way coupling) and nonlinearities are explicitly included in the forcing of the second linear system. First, we solve the linearized Euler equations for the strictly linear response to the same initial conditions (2.1)-(2.3) as 
in the Navier-Stokes computations. Defining

$$
\frac{\mathrm{D}}{\mathrm{D} t}=\frac{\partial}{\partial t}+\bar{u}_{j} \frac{\partial}{\partial x_{j}},
$$

the first set of linearized Euler equations can be written

$$
\begin{gathered}
\frac{\mathrm{D} u_{i}^{L}}{\mathrm{D} t}+u_{j}^{L} \frac{\partial \bar{u}_{i}}{\partial x_{j}}=-\frac{1}{\rho} \frac{\partial p^{L}}{\partial x_{i}} \\
\frac{\mathrm{D} p^{L}}{\mathrm{D} t}=-\gamma \bar{p} \frac{\partial u_{i}^{L}}{\partial x_{i}} .
\end{gathered}
$$

We have assumed that the time-dependent (but $x$-independent) base flow, denoted by variables with an overbar, has uniform pressure and is divergence-free. The time variation of the shear layer thickness parameter $\delta$ is taken from (2.9). Solved with a fourth-order Runge-Kutta time advance and second-order central differences in space, this provides the linear solution $\left(u_{i}^{L}, p^{L}\right)$, which is shown on figure $12(a)$ at time $t=250$ as contours of dilatation rate. This solution is the linear response of the thickening base flow to a localized disturbance in the initial condition. The contours show the dilatation rate associated with the wavepacket that develops from purely linear instability of the base flow.

An inhomogeneous linear problem is then solved to give the response $\left(u_{i}^{N}, p^{N}\right)$ to nonlinear forcing. In this case there are no initial disturbances and the response is driven by forcing terms $\left(f_{i}, q\right)$ on the right-hand side of the equations. The governing equations are

$$
\begin{gathered}
\frac{\mathrm{D} u_{i}^{N}}{\mathrm{D} t}+u_{j}^{N} \frac{\partial \bar{u}_{i}}{\partial x_{j}}=-\frac{1}{\rho} \frac{\partial p^{N}}{\partial x_{i}}+f_{i} \\
\frac{\mathrm{D} p^{N}}{\mathrm{D} t}=-\gamma \bar{p} \frac{\partial u_{i}^{N}}{\partial x_{i}}+q .
\end{gathered}
$$

At leading order the forcing terms according to the Lilley-Goldstein acoustic analogy (Lilley 1974; Goldstein 2001) are $q=0$ and

$$
f_{i}=-\frac{\partial u_{i}^{L} u_{j}^{L}}{\partial x_{j}},
$$

which thus introduces a quadratic nonlinearity. No further interactions of the two fields $\left(u_{i}^{L}, p^{L}\right)$ and $\left(u_{i}^{N}, p^{N}\right)$ are permitted. The model can be classified as the second term of an expansion in the amplitude of the perturbation and is also equivalent to the first step in a method of successive approximation (Van Dyke 1975).

The resulting dilatation rate for the nonlinearly driven field is shown on figure $12(b)$ at time $t=250$. The structure is quite different to the linear response. However, comparison with the Navier-Stokes solution at the same time (figure 12c) reveals similarities: the sound field has spread to a similar extent, indicating a similar time of origin, and the contour lobe patterns are qualitatively similar. The differences are due to the simplification of replacing the unsteady vortex packet with a prescribed base flow, and to higher-order nonlinear effects. We take this good qualitative agreement as a reason to study the response in more detail.

\subsection{Eigenmode-forced model}

In principle the linear wavepacket response shown on figure $12(a)$ can be reproduced using a sum of eigenmodes of the parallel flow stability problem, with the forcing 

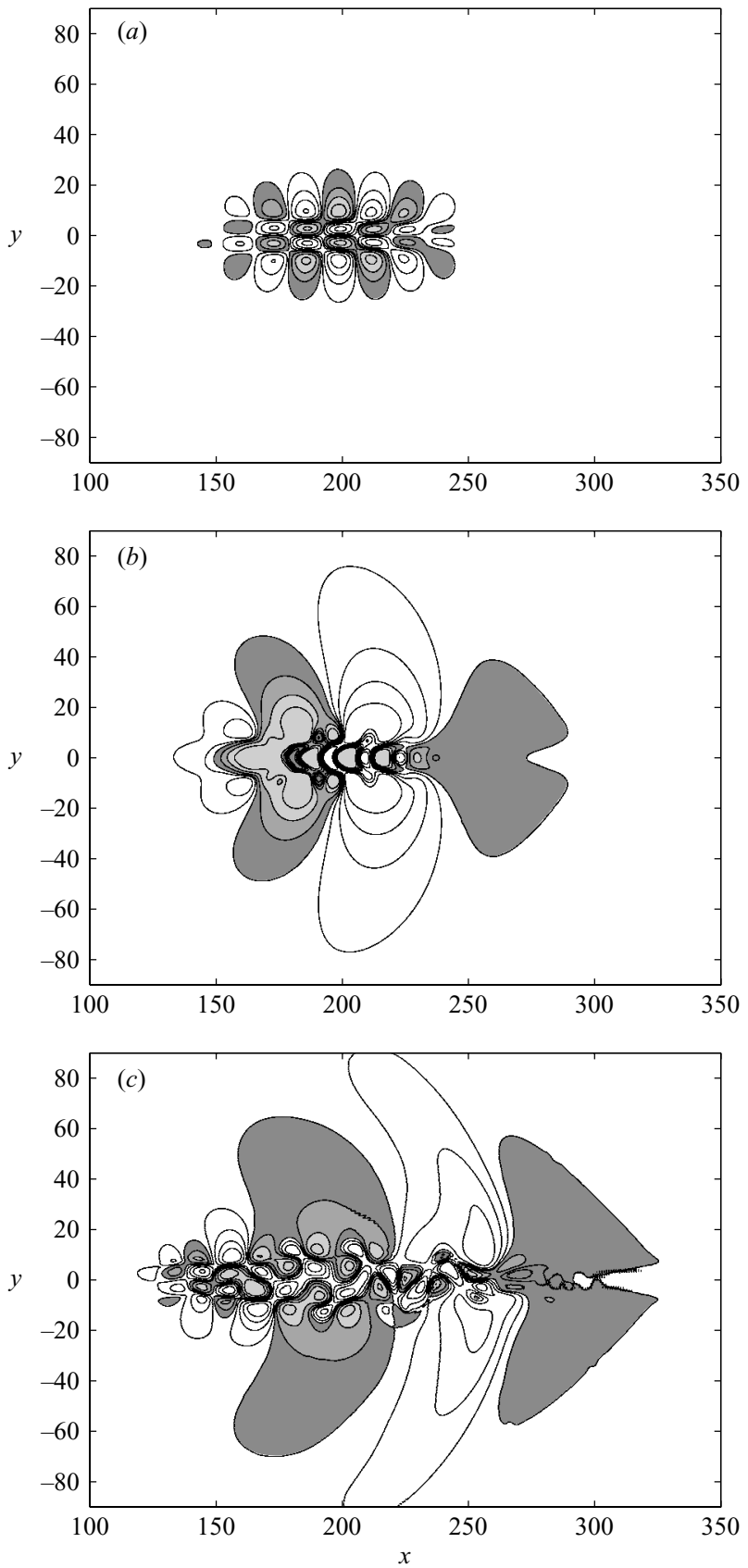

Figure 12. Dilatation rate contours at $t=250$. Contour levels $\pm c[0.05,0.20,0.40,0.65]$; filled contours are used for positive values, corresponding to areas of expansion: $(a)$ linear response to initial forcing $(c=20) ;(b)$ linear response to nonlinear forcing $(c=20000) ;(c)$ Navier-Stokes solution $(c=0.008)$.

constructed using eigenvalues and eigenfunctions from temporal linear stability theory. Such eigenmodes are defined by

$$
\phi=\hat{\phi}(y) \exp [\mathrm{i} k x+(\sigma-\mathrm{i} \omega) t],
$$


for variables $\phi=(u, v, p, T)$ with $k$ a wavenumber, $\omega$ a frequency, $\sigma$ a growth rate and $\hat{\phi}$ an eigenfunction containing the $y$-dependence. For high Reynolds numbers and relatively slow growth, the inviscid theory and local analysis will suffice. In this subsection we test a version of the two-step method of the previous subsection in which only the eigenvalues of the inviscid jet are required and the jet problem is reduced to a boundary-value problem. Forcing is applied at $y= \pm y_{0}$ corresponding to the locations of the inflection points in the jet mean profile, and is limited to a subset of the Lilley-Goldstein forcing terms. The success of this method in reproducing the more rigorous results from the previous coupled model will allow us to make a modal decomposition of the nonlinear forcing term (3.6).

In this simplified version we use a fixed base flow for the calculations (with $\delta=4$ chosen as a representative shear layer thickness). Outside the forcing layer the linearized governing equations reduce to the Pridmore-Brown (1958) (see also Goldstein 1976) equation

$$
\frac{\mathrm{D}}{\mathrm{D} t}\left[\frac{1}{\bar{c}^{2}} \frac{\mathrm{D}^{2} p}{\mathrm{D} t^{2}}-\nabla^{2} p+\frac{1}{\bar{\rho}} \frac{\partial \bar{\rho}}{\partial y} \frac{\partial p}{\partial y}\right]+2 \frac{\partial \bar{u}}{\partial y} \frac{\partial^{2} p}{\partial x \partial y}=0 .
$$

The forcing is chosen to represent the $u^{L} u^{L}$ component of the Reynolds stress forcing in (3.6). It is concentrated at $y= \pm y_{0}$ such that the jump relations for the forced Pridmore-Brown equation (derived in Appendix B) are satisfied, leading to the model boundary conditions

$$
p\left(y_{0}^{+}, t\right)-p\left(y_{0}^{-}, t\right)=0
$$

and

$$
\frac{\partial p}{\partial y}\left(y_{0}^{+}, t\right)-\frac{\partial p}{\partial y}\left(y_{0}^{-}, t\right)=\bar{\rho} \delta(t) \frac{\partial^{2} u u}{\partial x^{2}} .
$$

Here $\delta(t)$ is the time-dependent jet thickness parameter given by (2.9). Thus the time variation of jet thickness $\delta(t)$ is incorporated in the boundary condition (3.10), while the base flow $(\bar{u}, \bar{\rho})$ in $(3.8)$ is held fixed (based on $\delta=4)$. The Pridmore-Brown equation is solved for both $y \geqslant y_{0}$ and $y \leqslant y_{0}$ simultaneously, with the same pressure gradient boundary condition imposed at $y=y_{0}^{+}$and $y=y_{0}^{-}$. The pressure is updated at $y=y_{0}$ using the average update from $y=y_{0}^{+}$and $y=y_{0}^{-}$. This method imposes a normal pressure gradient jump condition and prevents the accumulation of a pressure jump at $y=y_{0}$ (consistent with (3.9)). This solution is then superposed with a solution where the lower shear layer $y_{0}=-5$ is forced in a similar manner.

To generate the nonlinear forcing term on the right of (3.10), an initial $u(x, t)=u_{0}(x)$ is specified with the same $x$ dependence as (2.3), and Fourier decomposed to give $\hat{u}_{0}(k)$. The forcing at $y_{0}=5$ is then generated from

$$
u(x, t)=\frac{1}{N} \sum_{k=1}^{N} \hat{u}_{0}(k) \exp (\mathrm{i} k x) \exp \left[\int(\sigma-\mathrm{i} \omega) \mathrm{d} t\right]+\text { c.c. }
$$

where c.c. denotes complex conjugate. The eigenvalues $\sigma$ and $\omega$ are interpolated from a database of results for the antisymmetric mode of inviscid linear instability of the base flow (using the code of Sandham \& Reynolds 1990 originally used for compressible mixing layers), parameterized with wavenumber $k$ and the shear layer thickness $\delta$.

To evaluate the usefulness of the eigenmode-forcing method, figure 13 compares the dilatation rate from this method with that of $\S 3.1$. Figure $13(a)$ is obtained from 

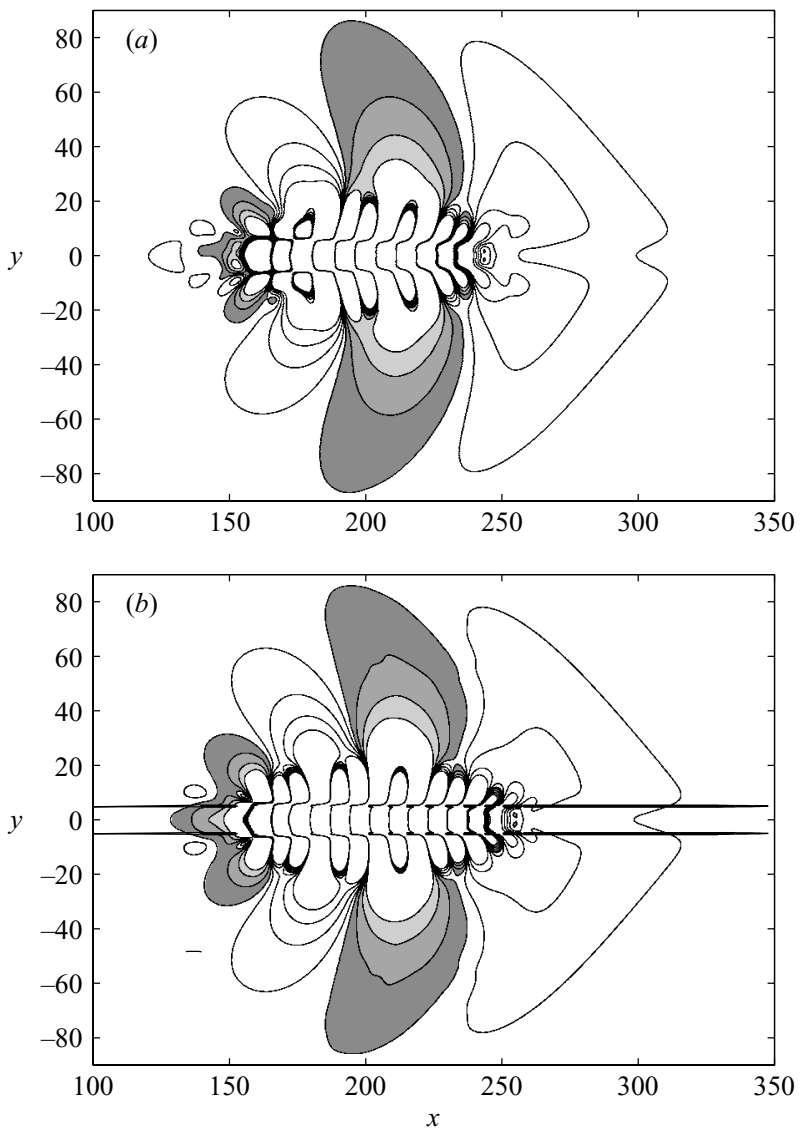

Figure 13. Dilatation rate contours at $t=250$. Contour levels $\pm c[0.05,0.20,0.40,0.65]$; filled contours are used for positive values, corresponding to areas of expansion: $(a)$ linear response to nonlinear forcing by $f_{x}$ only $(c=1500)$; $(b)$ equivalent linear response according to eigenmode forcing model $(c=10000)$.

a solution of the linearized Euler system subject to forcing by

$$
f_{x}=-\frac{\partial u^{L} u^{L}}{\partial x} .
$$

This is effectively the same term as given in (3.10), but distributed rather than concentrated at $y=y_{0}$; the extra $x$-derivative arises in (3.10) due to the fact that the forcing appears in a divergence form in the Pridmore-Brown equation (given in Appendix B). Figure $13(b)$ is the solution from the eigenmode method. The two are in very close correspondence, indicating that the local stability approximation, fixed base flow and confinement of disturbances to a plane are all acceptable simplifications of the linearized Euler model, for the purposes of replicating the main features of the sound field observed in the Navier-Stokes simulation.

\section{Discussion}

Before assembling an account of the sound production process in the model jet, we recap some results from the analysis of a modified wavy-wall problem. The traditional wavy-wall result in acoustics (e.g. Cremer \& Heckl 1988, Chap. VI, sections 4b 
and $6 \mathrm{~b}$ ) is that sound only radiates to the far field when the relative disturbance speed is supersonic. Sandham, Morfey \& Hu (2006) considered a modified wavywall problem in which the amplitude of a spatially periodic travelling wave grows, saturates over a controlled time scale and then decays, similarly to the Tam \& Morris (1980) and Crighton \& Huerre (1990) models. Numerical results were obtained over a wide range of the governing parameters, which are the convection Mach number, a dimensionless frequency (scaled on the initial growth rate) and a dimensionless saturation time. Classical cut-off phenomena were observed at low Mach number, at high frequency and for slow saturation time scale. Significant sound radiation at subsonic convection Mach numbers was demonstrated for sufficiently low frequencies and for sufficiently rapid saturation. This result can be interpreted in terms of the traditional wavy-wall problem by considering a time Fourier decomposition of the amplitude variation. After Fourier transformation there will be frequencies present which correspond to supersonic phase speeds of the travelling wave and hence can contribute to the sound radiation. In the following we focus on the acoustically active frequencies that are generated by nonlinear interactions of eigenmodes in the present jet noise model problem.

The nonlinear interaction term in the model of the previous subsection may be examined in more detail as follows. Two modes with wavenumbers $k_{i}$ and $k_{j}$ are considered, with the $i$ th linear mode decomposed as

$$
u_{i}^{L}=\hat{u}_{i} \exp \left(\mathrm{i} k_{i} x\right) \exp \left(\int\left(\sigma_{i}-\mathrm{i} \omega_{i}\right) \mathrm{d} t\right)+\text { c.c. }
$$

and similarly for the $j$ th mode. The nonlinear interaction term in (3.10) is then given by

$$
\frac{\partial^{2}\left(u_{i}^{L} u_{j}^{L}\right)}{\partial x^{2}}=A_{i j}^{+} \hat{u}_{i} \hat{u}_{j} \exp \left(\mathrm{i}\left(k_{i}+k_{j}\right) x\right)+A_{i j}^{-} \hat{u}_{i} \hat{u}_{j}^{\dagger} \exp \left(\mathrm{i}\left(k_{i}-k_{j}\right) x\right)+\text { c.c. }
$$

where ${ }^{\dagger}$ and c.c. denote complex conjugates, with prefactors for sum and difference mode interactions given by

$$
\begin{aligned}
& A_{i j}^{+}=-\left(k_{i}+k_{j}\right)^{2} \exp \left(\int\left(\sigma_{i}+\sigma_{j}-\mathrm{i}\left(\omega_{i}+\omega_{j}\right)\right) \mathrm{d} t\right), \\
& A_{i j}^{-}=-\left(k_{i}-k_{j}\right)^{2} \exp \left(\int\left(\sigma_{i}+\sigma_{j}-\mathrm{i}\left(\omega_{i}-\omega_{j}\right)\right) \mathrm{d} t\right) .
\end{aligned}
$$

By considering nonlinear interactions in this manner we can have strongly amplified disturbances with difference frequencies, $\omega_{i}-\omega_{j}$, that are small compared with the growth rate, $\sigma_{i}+\sigma_{j}$, and hence better coupled to sound in a subsonic jet.

Phase speeds arising from sum- and difference-mode interactions are given by

$$
\begin{gathered}
c_{i j}^{+}=\frac{\omega_{i}+\omega_{j}}{k_{i}+k_{j}}, \\
c_{i j}^{-}=\frac{\omega_{i}-\omega_{j}}{k_{i}-k_{j}} .
\end{gathered}
$$

For vanishing differences $k_{i}-k_{j}$ the difference-mode phase speed will tend to the group velocity. The phase speeds for all mode interactions are shown on figure 14 for all mode combinations up to integer wavenumber $\kappa=40(\kappa$ is related to $k$ by $\kappa=L_{x} k /(2 \pi)$, where $L_{x}=350$ is the length of the computational domain used in these calculations), at $t=200$. It can be seen that all the (two dimensional) interactions lead to subsonic phase speeds. 


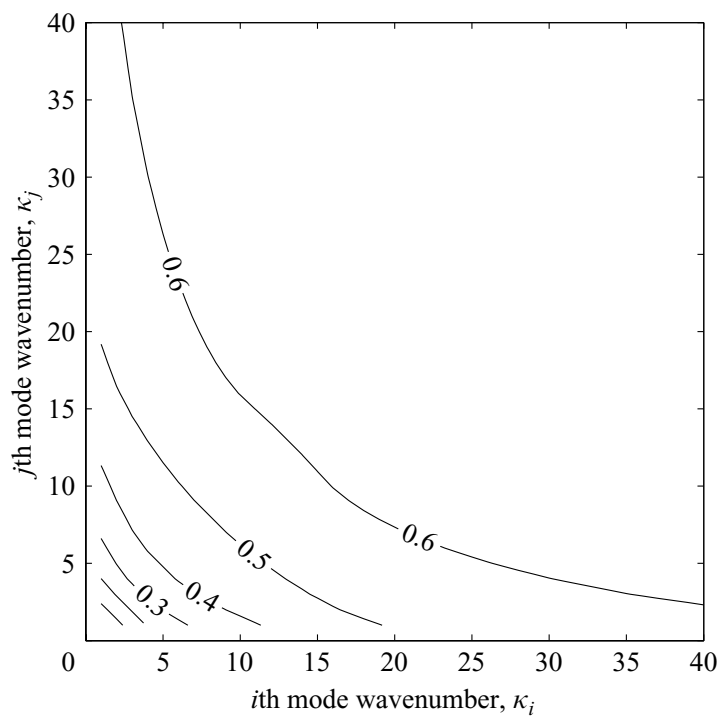

FIGURE 14. Phase speeds of quadratic difference mode interactions, scaled on jet centreline velocity.

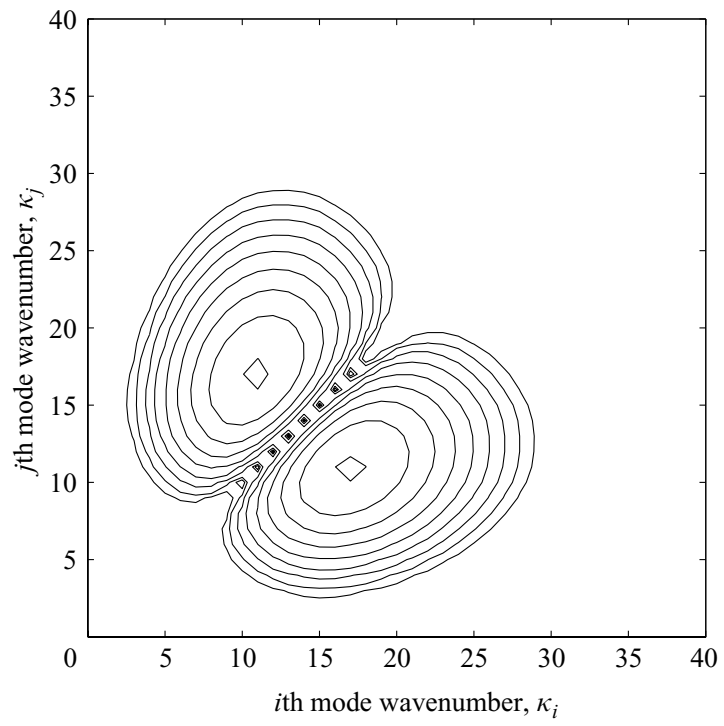

FIGURE 15. Contribution of (integer) difference wavenumbers $\kappa_{i}-\kappa_{j}$ to the eigenmode forcing model. Contour levels are at amplitudes $2^{m}$ with integer $m$.

Figure 15 shows contours of the real part of the difference-mode amplification factor $A_{i j}^{-}$. It can be seen that peaks occur at $\kappa=14 \pm 3$, where from figure 14 the phase speeds are in the range $0.55<c_{i j}^{-}<0.60$. Note that $\kappa=14$ gives a wavelength of 25, comparable with the scale of the linear response seen in figure 12(a); whereas the difference wavenumber $\Delta \kappa=6$ gives a wavelength of 58 , more representative of the sound field seen in figure $12(b)$. Based on $\Delta \kappa=6$ and an assumed phase speed of just under 0.6 , the period of the difference mode interaction is $T=100$. This may 
be compared to the period $T \approx 130$ seen in the computed sound radiation shown on figure 5 . The difference may be explainable from the wavy-wall model, in which the lower frequencies arising from smaller difference wavenumbers will be more effective radiators.

A summary of the proposed mechanism for the sound radiation seen in the Navier-Stokes simulations (e.g. figure $2 a$ ) is as follows. First, the mean flow is linearly unstable, and a broad band of wavenumbers (modes) is amplified. Secondly, as the most unstable of these waves reach high amplitude there is an exchange of energy with the mean flow, resulting in growth of the mean flow shear layer (via Reynolds stresses) and associated mode saturation. Simultaneously, nonlinear interactions will produce difference frequencies $\omega_{i}-\omega_{j}$ wavenumbers $k_{i}-k_{j}$ that radiate efficiently, despite their nominally subsonic phase speed. With this mechanism, $k_{i}-k_{j}$ wavenumber combinations from the most amplified linear instability modes can satisfy the necessary criteria of rapid saturation combined with frequencies that are small compared to the initial growth rate. The mechanism is not limited to a particular Mach number range.

This explanation based on nonlinear interactions of instability modes is consistent with the description of Chu \& Kovásznay (1958), who studied the bilinear interaction of vortical, acoustic and entropy modes in compressible flow. They observed that vortical-vortical interactions could give rise to sound, with a form of the source that was consistent with Lighthill (1952). The weakly nonlinear interactions studied here fall into this category since the primary instability mechanism is vortical. Quadratic nonlinearity of a similar type was also apparent in the experiments of Laufer \& Yen (1983), for forced vortex pairing in a round jet. They observed that the sound intensity varied as the fourth power of the near-field mode saturation amplitude, whereas a second power would be expected for a linear relationship of the type assumed in the Huerre \& Crighton (1983) model. In this connection we note that the difference-mode interaction between a fundamental $f$ and its subharmonic $f / 2$ is also $f / 2$, so the subharmonic radiation from vortex pairing can be fed by both a linear and weakly nonlinear mechanism.

The nonlinear interaction on the right-hand side of a linear wave equation has been emphasized up to this point, but we should note that strong nonlinearity also enters in the base flow, which thickens as the action of Reynolds stresses transfers energy from the mean flow into the disturbances. In the calculations shown here a variation of $\delta(t)$ was assumed, based on measurements from the Navier-Stokes calculations. Such a base flow variation could in principle be provided from a Reynolds-averaged Navier-Stokes (RANS) code. The nonlinear radiation model could then be used to predict those aspects of the sound field which can be attributed to interactions of large organized structures evolving on this base flow. By changing the mean flow one might be able to make the model responsive to detailed changes in nozzle design. We caution however that the local characteristics of instability wave saturation may well contribute to the sound radiation pattern; useful predictions may therefore require additional modelling of this nonlinear process.

\section{Conclusion}

The two-dimensional calculations presented here have demonstrated sound radiation from a perturbed two-dimensional plane jet flow, set up as an initial value problem. The scaling of the sound and its far-field characteristics suggest that the problem has some relevance to practical jet flows, while the nature of the model problem allows the sound origin to be located in time as well as space. Sound emission is observed 
to originate at an early stage of nonlinear evolution of the vortex packet that evolves from the primary asymmetric jet instability.

Two simplified acoustic models have been proposed. Both models solve a pair of linear systems, with the second system forced by quadratically nonlinear terms obtained from the first. Both models demonstrate a mechanism of sound generation by nonlinear interactions of primary instability modes. The second model employs linear eigenmodes and shows how the sound radiation is dominated by quadratic difference-wavenumber interactions between growing and decaying subsonic modes.

The authors acknowledge financial support from EPSRC under grant number GR/R46151.

\section{Appendix A. Numerical method}

The two-dimensional Navier-Stokes equations for a Newtonian fluid are written in dimensionless form using jet potential-core properties as reference values (density $\rho$, internal energy $e$ and pressure $p$ are respectively scaled on $\rho_{J}^{*}, U_{J}^{* 2}$ and $\rho_{J}^{*} U_{J}^{* 2}$ ), and $\delta_{\omega}^{*} / 2$ as reference length; the simulation Reynolds number (set to $R e=200$ ) is formed using these reference quantities. For this model problem the Prandtl number is set to $\operatorname{Pr}=1.0$. Note that in the following equations second derivatives are explicitly written for the $y$-direction, in which a finite difference scheme will be applied, to ensure that $2 h$ waves are damped ( $h$ being the grid spacing). The equations for mass, $x$ and $y$ momentum, and energy conservation are

$$
\begin{aligned}
& \frac{\partial \rho}{\partial t}=-\frac{\partial}{\partial x}(\rho u)-\frac{\partial}{\partial y}(\rho v), \\
& \frac{\partial \rho u}{\partial t}=\frac{\partial}{\partial x}\left(\tau_{x x}-\rho u^{2}-p\right)+\frac{\partial}{\partial y}\left(\frac{\mu}{R e} \frac{\partial v}{\partial x}-\rho u v\right)+\frac{\mu}{R e} \frac{\partial^{2} u}{\partial y^{2}}+\frac{1}{R e} \frac{\partial u}{\partial y} \frac{\partial \mu}{\partial y}, \\
& \frac{\partial \rho v}{\partial t}=\frac{\partial}{\partial x}\left(\tau_{x y}-\rho u v\right)+\frac{\partial}{\partial y}\left(-\rho v^{2}-p-\frac{2}{3} \frac{\mu}{R e} \frac{\partial u}{\partial x}\right)+\frac{\mu}{R e} \frac{4}{3} \frac{\partial^{2} v}{\partial y^{2}}+\frac{1}{R e} \frac{4}{3} \frac{\partial v}{\partial y} \frac{\partial \mu}{\partial y}, \\
& \frac{\partial \rho E}{\partial t}=\frac{\partial}{\partial x}\left(u \tau_{x x}+v \tau_{x y}-u(\rho E+p)+\frac{\gamma \mu}{\operatorname{Pr} R e} \frac{\partial e}{\partial x}\right) \\
& +\frac{\partial}{\partial y}\left(\frac{u \mu}{\operatorname{Re}} \frac{\partial v}{\partial x}-\frac{v \mu}{\operatorname{Re}} \frac{2}{3} \frac{\partial u}{\partial x}-v(\rho E+p)\right)+\frac{\gamma \mu}{\operatorname{RePr}} \frac{\partial^{2} e}{\partial y^{2}}+\frac{\gamma}{\operatorname{RePr}} \frac{\partial e}{\partial y} \frac{\partial \mu}{\partial y} \\
& +\frac{u \mu}{R e} \frac{\partial^{2} u}{\partial y^{2}}+\frac{\partial u}{\partial y} \frac{\partial}{\partial y}\left(\frac{u \mu}{R e}\right)+\frac{4}{3} \frac{v \mu}{R e} \frac{\partial^{2} v}{\partial y^{2}}+\frac{4}{3} \frac{\partial v}{\partial y} \frac{\partial}{\partial y}\left(\frac{v \mu}{R e}\right) .
\end{aligned}
$$

The stresses are given by

$$
\begin{gathered}
\tau_{x x}=\frac{\mu}{R e}\left(\frac{4}{3} \frac{\partial u}{\partial x}-\frac{2}{3} \frac{\partial v}{\partial y}\right), \\
\tau_{x y}=\frac{\mu}{R e}\left(\frac{\partial u}{\partial y}+\frac{\partial v}{\partial x}\right),
\end{gathered}
$$

and the total energy is given by

$$
E=e+\frac{1}{2}\left(u^{2}+v^{2}\right)
$$


where the internal energy $e$ is related to pressure $p$ and density $\rho$ by the equation of state for a perfect gas

$$
p=(\gamma-1) \rho e .
$$

The viscosity $\left(\mu=\mu^{*} / \mu_{J}^{*}\right)$ is given by a power law

$$
\mu=T^{0.67},
$$

and the temperature is related to the internal energy by

$$
T=\gamma(\gamma-1) M^{2} e .
$$

Periodic boundary conditions are applied in the $x$-direction, with derivatives in that direction evaluated using Fourier methods. In the $y$-direction we employ a symmetric sixth-order compact finite difference scheme, based on a tridiagonal left-hand-side matrix and a pentadiagonal right-hand side; see Lele (1992). A stretched grid is employed for the $y$-direction, with coordinates given by

$$
y=L_{y} \frac{\sinh \left(c_{y} \eta\right)}{\sinh c_{y}}
$$

where $\eta$ is uniformly spaced in $[-1,1]$. The computational box thus extends to $\pm L_{y}$. First and second derivatives of any function $f$ are computed by

$$
\begin{gathered}
f_{y}=\frac{f_{\eta}}{y_{\eta}}, \\
f_{y y}=\frac{f_{\eta \eta}-y_{\eta \eta} f_{y}}{y_{\eta}^{2}},
\end{gathered}
$$

where a subscript denotes differentiation. In all the calculations presented, a stretching factor $c_{y}=1.5$ has been used.

Standard characteristic boundary conditions (Thompson 1987) are applied at $y= \pm L_{y} / 2$. Due to the initial-value nature of the problem studied in this paper, the simulations do not need to be run to long times and boundary reflections are not an issue (this is one of the benefits of the approach taken).

The computational grid is fine enough to ensure that all relevant flow features are resolved. The solution is time advanced using a third-order Runge-Kutta method, with a fixed time step. In the Fourier $(x)$ direction it is necessary to explicitly filter the 'oddball' wavenumber $\kappa_{x}=N_{x} / 2$ so that $2 \delta$ oscillations do not build up. A filtered right-hand side $\hat{R}_{i}^{m}$ for $m=1 \ldots 4$, representing (A 1)-(A 4) at a grid point $i$, is given in terms of the original $R_{i}^{m}$ by

$$
\hat{R}_{i}^{m}=R_{i}^{m}-\frac{(-1)^{i}}{N_{x}} \sum_{j=1}^{N_{x}}(-1)^{j} R_{j}^{m} .
$$

This is applied at every sub-step of the time advance.

\section{Appendix B. Jump relations for the forced Pridmore-Brown equation}

In $\S 3.2$ we replace the acoustic forcing caused by hydrodynamic fluctuations distributed through a shear layer with forcing on a surface. To enable a consistent choice of boundary conditions we consider jump relations of the third-order Pridmore-Brown wave equation. The basic problem is specified in two space dimensions $(x, y)$ for a fixed base flow, with velocity $\bar{u}$, density $\bar{\rho}$ and sound speed $\bar{c}$ being functions of $y$ 
alone. With forcing terms $f_{x}, f_{y}$ and $q$ included from the Lilley-Goldstein analogy, the inhomogeneous Pridmore-Brown equation can be written in the form

$$
\mathrm{L}(p)=\bar{\rho} Q
$$

with the linear operator

$$
\mathrm{L}(p)=\frac{\mathrm{D}}{\mathrm{D} t}\left[\frac{1}{\bar{c}^{2}} \frac{\mathrm{D}^{2} p}{\mathrm{D} t^{2}}-\frac{\partial^{2} p}{\partial x^{2}}-\frac{\partial^{2} p}{\partial y^{2}}+\frac{1}{\bar{\rho}} \frac{\partial \bar{\rho}}{\partial y} \frac{\partial p}{\partial y}\right]+2 \frac{\partial \bar{u}}{\partial y} \frac{\partial^{2} p}{\partial x \partial y}
$$

and forcing term

$$
Q=\frac{\mathrm{D}^{2} q}{\mathrm{D} t^{2}}-\frac{\mathrm{D}}{\mathrm{D} t}\left(\frac{\partial f_{x}}{\partial x}+\frac{\partial f_{y}}{\partial y}\right)+2 \frac{\partial \bar{u}}{\partial y} \frac{\partial f_{y}}{\partial x} .
$$

The substantial derivative is given by

$$
\frac{\mathrm{D}}{\mathrm{D} t}=\frac{\partial}{\partial t}+\bar{u} \frac{\partial}{\partial x}
$$

The forcing inputs $f_{x}, f_{y}$ and $q$ are now taken to be confined to the plane $y=y_{0}$; we write

$$
f_{x}=\hat{f}_{x}(x, t) \delta\left(y-y_{0}\right),
$$

and similarly for $f_{y}$ and $q$. In general the pressure $p$ and its normal derivative $\partial p / \partial y$ will be discontinuous at $y=y_{0}$. The jump relations are found by integrating (B 1)-(B 3) across $y=y_{0}$. We define

$$
\Delta p=p\left(y_{0}^{+}\right)-p\left(y_{0}^{-}\right)
$$

and

$$
\Delta p^{\prime}=\frac{\partial p}{\partial y}\left(y_{0}^{+}\right)-\frac{\partial p}{\partial y}\left(y_{0}^{-}\right) .
$$

Integration of (B 2) from $y_{0}^{-}$to $y_{0}^{+}$gives

$$
\int \mathrm{L}(p) \mathrm{d} y=\frac{\mathrm{D}}{\mathrm{D} t}\left(-\Delta p^{\prime}+\frac{1}{\bar{\rho}} \frac{\partial \bar{\rho}}{\partial y} \Delta p\right)+2 \frac{\partial \bar{u}}{\partial y} \frac{\partial(\Delta p)}{\partial x}
$$

and integration again gives

$$
\iint \mathrm{L}(p) \mathrm{d} y^{\prime} \mathrm{d} y=-\frac{\mathrm{D}(\Delta p)}{\mathrm{D} t} .
$$

The same procedure applied to (B 3) gives

$$
\int Q \mathrm{~d} y=\frac{\mathrm{D}^{2} \hat{q}}{\mathrm{D} t^{2}}-\frac{\mathrm{D}}{\mathrm{D} t}\left(\frac{\partial \hat{f}_{x}}{\partial x}\right)+2 \frac{\partial \bar{u}}{\partial y} \frac{\partial \hat{f}_{y}}{\partial x}
$$

and

$$
\iint Q \mathrm{~d} y^{\prime} \mathrm{d} y=-\frac{\mathrm{D} \hat{f}_{y}}{\mathrm{D} t} .
$$

Substitution of (B 9) and (B 11) into (B 1) gives the pressure jump relation

$$
\Delta p=\bar{\rho} \hat{f}_{y} .
$$


A pressure gradient jump relation is found by substituting (B 8) and (B 10) into (B 1) and eliminating the pressure jump using (B 12), leading to

$$
\frac{\mathrm{D}\left(\Delta p^{\prime}\right)}{\mathrm{D} t}=-\bar{\rho}\left[\frac{\mathrm{D}^{2} \hat{q}}{\mathrm{D} t^{2}}-\frac{\mathrm{D}}{\mathrm{D} t}\left(\frac{\partial \hat{f}_{x}}{\partial x}\right)\right]+\frac{\partial \bar{\rho}}{\partial y} \hat{f}_{y} .
$$

For the special case of a constant-density shear flow this reduces to

$$
\Delta p^{\prime}=\bar{\rho}\left(\frac{\partial \hat{f}_{x}}{\partial x}-\frac{\mathrm{D} \hat{q}}{\mathrm{D} t}\right) .
$$

Equations (B 12) and (B 14) constitute the jump relations for a constant-density shear flow. In $\S 3.2$ we apply these equations for one active forcing term $\hat{f}_{x}$, for which $p$ is continuous but there is a jump in $\partial p / \partial y$ that can be imposed as a boundary condition.

\section{REFERENCES}

Brown, G. L. \& Roshko, A. 1974 On density effects and large tructure in turbulent mixing layers. J. Fluid Mech. 64, 775-816.

Chu, B.-T. \& Kovásznay, L. S. 1958 Non-linear interactions in a viscous heat-conducting compressible gas. J. Fluid Mech. 3, 494-514.

Colonius, T. 2004 Modelling artificial boundary conditions for compressible flow. Annu. Rev. Fluid Mech. 36, 315-345.

Colonius, T., Lele, S. K. \& Moin, P. 1997 Sound generation in a mixing layer. J. Fluid Mech. 330, $375-409$.

Cremer, L. \& Heckl, M. 1988 Structure-Borne Sound (2nd edn transl. and revised by E. E. Ungar) Springer.

Crighton, D. G. 1975 Basic principles of aerodynamic noise generation. Prog. Aerospace Sci. 16(1), 31-96.

Crighton, D. G. \& Huerre, P. 1990 Shear-layer pressure fluctuations and superdirective acoustic sources. J. Fluid Mech. 220, 355-368.

Ffowcs Williams, J. E. \& Hawkings, D. L. 1969 Sound generation by turbulence and surfaces in arbitrary motion. Phil. Trans. R. Soc. Lond. A 264, 321-342.

FreUND, J. B. 2001 Noise sources in a low-Reynolds-number turbulent jet at Mach 0.9. J. Fluid Mech. 438, 277-305.

Goldstein, M. E. 1976 Aeroacoustics. McGraw-Hill.

Goldstein, M. E. 2001 An exact form of Lilley's equation with a velocity quadrupole/temperature dipole source term. J. Fluid Mech. 443, 231-236.

Howe, M. S. 1970 Transmission of an acoustic pulse through a plane vortex sheet. J. Fluid Mech. 43, 353-367.

Hu, Z. W., Morfey, C. L. \& Sandham, N. D. 2002 Sound radiation from a subsonic plane jet. AIAA Paper 2002-2421.

Hu, Z. W., Morfey, C. L. \& Sandham, N. D. 2003 Sound radiation in turbulent channel flows. J. Fluid Mech. 475, 269-302.

Huerre, P. \& Crighton, D. G. 1983 Sound generation by instability waves in a low Mach number jet. AIAA Paper 83-0661.

LAUfer, J. \& Yen, T.-C. 1983 Noise generation by a low-mach-number jet. J. Fluid Mech. 134, 1-31.

LeLE, S. K. 1992 Compact finite diffrerence schemes with spectral-like resolution. J. Comput. Phys. $103,16-42$.

Lighthill, M. J. 1952 On sound generated aerodynamically: I. General theory. Proc. R. Soc. Lond. A 222, 564-587.

LILLEY, G. M. 1974 On the noise from jets. AGARD CP-131, pp 13.1-13.12.

LOCKARD, D. P. 2000 An efficient, two-dimensional implementation of the Ffowcs Williams and Hawkings equation. J. Sound Vib. 229(4), 897-911. 
Lush, P. A. 1971 Measurements of subsonic jet noise and comparison with theory. J. Fluid Mech. 46, 477-500.

Mitchell, B. E., Lele, S. K. \& Moin, P. 1999 Direct computation of the sound generated by vortex pairing in an axisymmetric jet. J. Fluid Mech. 383, 113-142.

Morris, P. J., Giridharan, M. G. \& Lilley, G. M. 1990 On the turbulent mixing of compressible free shear layers. Proc. R. Soc. Lond. A 431, 219-243

Pridmore-Brown, D. C. 1958 Sound propagation in a fluid flowing through an attenuating duct. J. Fluid Mech. 4, 393-406.

Sandham, N. D., Morfey, C. L. \& Hu, Z. W. 2006 Sound radiation from exponentially growing and decaying surface waves. J. Sound Vib. 294, 355-361.

Sandham, N. D. \& Reynolds, W. C. 1990 Compressible mixing layer: linear theory and direct simulation. AIAA J. 28(4), 618-624.

Shur, M. L., Spalart, P. R. \& Strelets, M. K. 2005 Noise prediction for increaslingly complex jets. Part 1: Methods and tests. Intl J. Aeroacoust. 4, 213-246.

SuzuKI, T. \& Colonius, T. 2006 Instability waves in a subsonic round jet detected using a near-field phased microphone array. J. Fluid Mech. 565, 197-226.

TAM, C. K. W. \& Morris, P. J. 1980 The radiation of sound by the instability waves on a compressible plane turbulent shear layer. J. Fluid Mech. 98, 349-381.

Tester, B. J. \& Morfey, C. L. 1976 Developments in jet noise modelling - theoretical predictions and comparisons with measured data. J. Sound Vib. 46(1), 79-103.

Thompson, K. W. 1987 Time dependent boundary conditions for hyperbolic systems. J. Comput. Phys. 68, 1-24.

Van Dyke, M. 1975 Perturbation Methods in Fluid Mechanics. The Parabolic Press, Stanford, California. 